\section{RSP}

http://www.rsp.fsp.usp.br/
Revista de Saúde Pública

\title{
Nascer no Brasil: a presença do acompanhante favorece a aplicação das boas práticas na atenção ao parto na região Sul
}

\author{
Juliana Jacques da Costa Monguilhott', Odaléa Maria Brüggemann", Paulo Fontoura Freitas'"', \\ Eleonora d'Orsi ${ }^{\mathrm{iv}}$ \\ I Instituto Federal de Educação, Ciência e Tecnologia de Santa Catarina. Departamento Acadêmico de Saúde e \\ Serviços. Florianópolis, SC, Brasil \\ " Universidade Federal de Santa Catarina. Programa de Pós-Graduação em Enfermagem. Florianópolis, SC, Brasil \\ II' Universidade do Sul de Santa Catarina. Núcleo de Orientação em Epidemiologia. Curso de Medicina. Palhoça, \\ SC, Brasil \\ Iv Universidade Federal de Santa Catarina. Departamento de Saúde Pública. Programa de Pós-Graduação em \\ Saúde Coletiva. Florianópolis, SC, Brasil
}

\section{RESUMO}

OBJETIVO: Analisar se a presença do acompanhante favorece a aplicação das boas práticas na atenção ao parto na região Sul do Brasil.

MÉTODOS: Análise transversal do estudo longitudinal Nascer no Brasil. Foram analisados dados de 2.070 mulheres da região Sul que entraram em trabalho de parto. Os dados foram coletados entre fevereiro e agosto de 2011, por meio de entrevista e prontuário. Realizou-se análise bivariada e multivariada, calculando-se razões de prevalência brutas e ajustadas por regressão de Poisson com estimação de variância robusta. Adotou-se nível de significância de 5\%.

RESULTADOS: A maioria das mulheres teve o acompanhante durante o trabalho de parto $(51,7 \%)$, mas poucas permaneceram com ele no parto $(39,4 \%)$ ou na cesariana $(34,8 \%)$. Menos da metade das mulheres teve acesso às várias práticas recomendadas, enquanto práticas não recomendadas continuam sendo realizadas. No modelo ajustado por idade, escolaridade, fonte de pagamento do parto, paridade e escore da Associação Brasileira de Institutos de Pesquisa de Mercado, a presença do acompanhante esteve estatisticamente associada à maior oferta de líquidos/alimentos $(\mathrm{RPa}=1,34)$, prescrição de dieta $(\mathrm{RPa}=1,34)$, uso de métodos não farmacológicos para alívio da dor $(\mathrm{RPa}=1,37)$, amniotomia $(\mathrm{RPa}=1,10)$, analgesia peridural ou ráqui $(\mathrm{RPa}=1,84)$, adoção de posição não litotômica no parto $(\mathrm{RPa}=1,77)$, permanência na mesma sala durante o trabalho de parto, parto e pós-parto $(\mathrm{RPa}=1,62)$, contato pele a pele no parto $(\mathrm{RPa}=1,81)$ e na cesariana $(\mathrm{RP}=2,43)$, bem como redução da manobra de Kristeller $(\mathrm{RPa}=0,67)$, tricotomia $(\mathrm{RPa}=0,59)$ e enema $(\mathrm{RPa}=0,49)$.

CONCLUSÕES: Na região Sul do Brasil, além de sofrer várias intervenções desnecessárias, a maioria das mulheres não têm acesso às boas práticas. A presença do acompanhante está associada a diversas práticas benéficas e à redução de algumas intervenções, embora outras não sofram impacto.

DESCRITORES: Parto Humanizado. Humanização da Assistência. Direitos do Paciente. Prática Clínica Baseada em Evidências. Serviços de Saúde Materno-Infantil. 


\section{INTRODUÇÃO}

O modelo de assistência à saúde fragmentado, curativo e hospitalar caracterizou toda a construção do sistema de saúde do Brasil, de forma a privilegiar e consolidar as práticas médicas individuais, financiadas pelo sistema previdenciário, em detrimento das ações coletivas de prevenção e promoção da saúde. Nesse cenário, a obstetrícia moderna e o modelo tecnocrático de assistência ao parto se legitimaram com facilidade e o evento complexo do parto e nascimento foi separado da vida familiar e comunitária para se tornar um assunto médico e hospitalar ${ }^{23}$. A adoção de práticas obstétricas que surgiram com a institucionalização do parto, afim de controlar o processo fisiológico do nascimento e racionalizar padrões de trabalho, caracterizaram gradativamente o processo parturitivo como patológico e negaram a capacidade de parir intrínseca à mulher .

De evento fisiológico, familiar e social, o parto/nascimento transforma-se em ato médico, no qual o risco de patologias e complicações se torna a regra e não a exceção ${ }^{23}$. Nesse modelo brasileiro, percebe-se a utilização de práticas que são consideradas claramente prejudiciais pela Organização Mundial da Saúde ${ }^{30}$ (OMS) e que não possuem evidências científicas para a sua utilização, como o uso rotineiro de cateter venoso periférico $(74,9 \%)$, dieta zero $(74,8 \%)$, posição de litotomia $(91,7 \%)$, uso rotineiro de ocitócitos $(36,4 \%)$ e amniotomia $(39,1 \%)$, manobra de Kristeller $(36,1 \%)$ e número excessivo de episiotomias $(53,5 \%)$. Contudo, as boas práticas que devem ser estimuladas não são implementadas plenamente, como o uso de métodos não farmacológicos (MNF) para o alívio da dor $(26,7 \%)$ e o respeito à presença contínua do acompanhante de escolha da mulher $(18,8 \%)^{10,21}$. Considerando que o conceito de boas práticas na assistência ao parto ainda não está descrito na literatura, neste estudo, elas são entendidas como a adoção das recomendações da $\mathrm{OMS}^{30}$, a implementação das evidências científicas e a eliminação das intervenções desnecessárias.

O Ministério da Saúde tem buscado qualificar a assistência ao parto e nascimento e apoiar a implementação das evidências científicas por meio das políticas públicas de saúde. Dessas políticas, destaca-se atualmente a Rede Cegonha ${ }^{25}$, que objetiva garantir às mulheres e aos recém-nascidos a realização de parto e nascimento seguros, incluindo o apoio por acompanhante de livre escolha da mulher.

Em revisão sistemática sobre o apoio durante o nascimento, as mulheres que recebem apoio contínuo durante o parto apresentam maior probabilidade de ter um parto vaginal espontâneo e trabalho de parto menos prolongado; menor probabilidade de serem submetidas a analgesia intraparto/anestesia, parto vaginal instrumental ou cesariana; e de ter um bebê com baixo índice de Apgar no quinto minuto de vida ${ }^{17}$.

Em ensaio clínico randomizado realizado no Brasil, as parturientes com acompanhante de sua escolha tiveram maior satisfação global com a experiência do trabalho de parto e parto do que as do grupo controle. Entretanto, é necessário avaliar a influência do acompanhante na adoção de boas práticas na atenção ao parto ${ }^{3}$. Em pesquisas qualitativas, as mulheres referem que o acompanhante fornece segurança e o apoio emocional necessário, por palavras e gestos de carinho e conforto ${ }^{29,31}$. Há relatos de que o apoio do acompanhante, associado a boas práticas como o banho e a movimentação, torna-se um fator capaz de reduzir a dor e a duração do trabalho de parto ${ }^{5,35}$.

(BR), Casa Civil, Subchefia para Assuntos Jurídicos. Lei $\mathrm{N}^{\circ} 11.108$, de 7 de abril de 2005. Altera a Lei no 8.080, de 19 de setembro de 1990, para garantir às parturientes o direito à presença de acompanhante durante o trabalho de parto, parto e pós-parto imediato, no âmbito do Sistema Único de Saúde - SUS. Brasília (DF); 2005 [citado 16 jun 2017]. Disponível em: http://www.planalto.gov.br/ ccivil_03/_ato2004-2006/2005/ lei/l11108.htm

O Ministério da Saúde brasileiro reconhece os benefícios dessa prática e a publicação da Lei 11.108, em 2005a garante às parturientes o direito à presença de acompanhante durante o trabalho de parto, parto e pós-parto imediato, no âmbito do Sistema Único de Saúde (SUS), incluindo rede própria ou conveniada. Apesar disso, muitas mulheres ainda são privadas desse direito. Dados gerais da pesquisa Nascer no Brasil sugerem que as mulheres brasileiras estão sendo expostas aos riscos de iatrogenia no parto e que, apesar de ser considerado um marcador de segurança e qualidade do atendimento, a presença contínua de um acompanhante ainda não é garantida para a maioria delas $(81,2 \%)^{10}$. 
Dessa forma, o objetivo desta pesquisa foi analisar se a presença do acompanhante favorece a aplicação das boas práticas na atenção ao parto na região Sul do Brasil.

\section{MÉTODOS}

Análise transversal de um estudo longitudinal, realizada a partir da pesquisa: "Nascer no Brasil: inquérito nacional sobre parto e nascimento". Tal estudo considerou elegíveis todas as instituições de saúde do Brasil que registraram ao menos 500 partos/ano segundo o Sistema de Informações sobre Nascidos Vivos (Sinasc), 2007b. Foram incluídas as puérperas hospitalizadas por motivo de parto que tivessem como produto da concepção recém-nascidos com 22 semanas gestacionais ou mais, ou mais de $500 \mathrm{~g}$ de peso ao nascer, sendo excluídas as puérperas cujo parto tivesse ocorrido no domicílio, no transporte para a maternidade ou em outra unidade de saúde que não a selecionada ${ }^{20}$.

A coleta de dados foi realizada em 2011 e 2012 por meio de entrevistas utilizando formulários eletrônicos. O primeiro questionário foi preenchido durante entrevistas com as puérperas dentro das primeiras 24 horas após o nascimento. O segundo questionário foi preenchido com dados disponíveis nos prontuários maternos, após a alta do hospital ou no $42^{\circ}$ dia para a mulher e no $28^{\circ}$ dia para o recém-nascido que permaneceram internados. Participaram 50 supervisores e 200 entrevistadores, constituindo 27 equipes estaduais. Informações detalhadas sobre a coleta de dados estão descritas em outra publicação ${ }^{20}$.

O tamanho amostral foi de 90 puérperas e seus conceptos para cada instituição de saúde sorteada, sendo representativa por região do Brasil. A amostra foi estratificada por macrorregião geográfica, tipo de município (capital/interior) e administração hospitalar (pública, mista ou privada). O número de mulheres amostrado foi 23.940, distribuídas em 191 municípios em todos os estados do país. O tamanho da amostra em cada estrato foi calculado com base na taxa de cesariana no Brasil em 2007, de 46,6\%, com significância de $5 \%$ para detectar diferenças de $14 \%$ (diferença entre hospitais mistos e hospitais privados) com poder de $80 \%$. O desenho da amostra Nascer no Brasil foi descrito com detalhes por Vasconcellos et al..$^{40}$

Para o presente estudo foram selecionados os dados da região Sul do Brasil, coletados entre fevereiro e agosto de 2011, em 46 instituições de saúde sorteadas (17 do Paraná, 13 de Santa Catarina e 16 do Rio Grande do Sul), compondo uma amostra de 4.139 mulheres. Foram incluídas aquelas que entraram em trabalho de parto (espontâneo ou induzido), totalizando uma amostra de 2.070 mulheres. Considerou-se em trabalho de parto mulheres que atingiram dilatação cervical de quatro centímetros ou mais.

Os dados utilizados no presente estudo emergiram de dois instrumentos: questionário da entrevista hospitalar com a puérpera e instrumento para coleta de dados do prontuário materno.

Cada prática obstétrica foi considerada como uma variável de desfecho. As variáveis de desfecho selecionadas a partir da entrevista com a puérpera foram: oferecimento de líquidos ou alimentos (sim; não), livre movimentação (sim; não), uso de MNF de alívio da dor (sim; não, considerando banheira; chuveiro; bola; massagem; banquinho de cócoras; cavalinho), amniotomia (sim; não), tricotomia (sim; não), enema (sim; não), posição não litotômica (sim - deitada de lado; sentada/reclinada; na banheira; quatro apoios; cócoras; em pé; não litotomia;), manobra de Kristeller (sim; não), sala PPP - pré-parto/parto/pós parto (sim; não) e contato pele a pele (sim; não). As variáveis de desfecho selecionadas a partir do prontuário

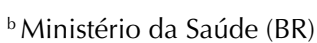
Sistema de Informação da Atenção Básica -SIAB. Brasília (DF); 2008 [citado 16 jun 2017]. Disponível em: http://www2. datasus.gov.br/SIAB/index. php?area $=04$ A0 1 \&item $=1$ materno foram: prescrição de dieta (sim; não); prescrição de ocitocina (sim; não), analgesia peridural ou ráqui (sim; não); episiotomia (sim; não).

As duas variáveis principais de exposição foram: acompanhante no trabalho de parto e acompanhante no parto, sendo construídas de forma composta, com dados da entrevista e com dados do prontuário materno. 
Para testar a associação entre a presença do acompanhante e as boas práticas (desfechos) na assistência ao trabalho de parto e parto, foram construídos modelos multivariados (um para cada desfecho), ajustados por cinco variáveis identificadas na literatura ${ }^{21}$. As variáveis de ajuste, selecionadas a partir da entrevista com a puérpera, foram: idade (de 12 a 19; de 20 a 34 anos; 35 ou mais), escolaridade (ensino fundamental incompleto; ensino fundamental completo; ensino médio; ensino superior completo ou mais) e escore socioeconômico ABIPEME - Associação Brasileira de Institutos de Pesquisa de Mercado (classe A+B; classe C; classe D+E). As variáveis de ajuste selecionadas a partir dos dados do prontuário materno foram: paridade (primípara; multípara) e fonte de pagamento do parto (pública; privada). A hipótese estudada foi de que a presença do acompanhante favorece as boas práticas.

O critério de classificação econômica adotado para gerar a variável ABIPEME foi o preconizado pela Associação Brasileira de Empresas de Pesquisa (ABEP), que estima o poder de compra das pessoas e famílias urbanas, baseado na posse de bens e no grau de instrução do chefe da família ${ }^{15}$.

O programa estatístico utilizado foi o Stata/SE, versão 11. Foi aplicada estatística descritiva e o teste do qui-quadrado utilizado na comparação de proporções. Para estimar as razões de prevalência brutas e ajustadas e seus respectivos intervalos de confiança de 95\% (IC95\%), foi utilizada regressão de Poisson com variância robusta. As variáveis que entraram no modelo ajustado foram consideradas pertencentes ao mesmo nível hierárquico. Foram considerados significativos valores de $\mathrm{p}<0,05$.

Esta investigação está norteada pela Portaria 196/96 do Conselho Nacional de Saúde, que dispõe sobre as diretrizes e normas da pesquisa com seres humanos (CEP/ENSP - Protocolo 92/10). Todas as puérperas assinaram o termo de consentimento livre e esclarecido.

\section{RESULTADOS}

As características sociodemográficas e obstétricas das participantes que entraram em trabalho de parto e, portanto, foram incluídas neste estudo $(n=2.070)$, estão apresentadas na Tabela 1. A maioria das mulheres estava incluída na faixa etária de 20 a 34 anos $(67,8 \%)$, pertencia à classe socioeconômica C (60,3\%), era multípara $(57,0 \%)$, realizou parto vaginal (85,0\%), teve o parto no setor público $(89,7 \%)$ e permaneceu com o acompanhante durante o trabalho de parto (51,7\%). Entretanto, menos da metade teve a presença do acompanhante durante o parto $(39,4 \%)$ ou no momento da cesariana (34,8\%). Quanto à escolaridade, poucas mulheres concluíram o ensino superior (5,7\%).

Em relação às práticas obstétricas realizadas durante o trabalho de parto, menos da metade das mulheres foram autorizadas a beber ou comer nesse período (32,7\%). A maioria delas pôde movimentar-se (59,2\%), mas poucas fizeram uso de algum MNF para alívio da dor (32,7\%) banheira, chuveiro, bola, massagem, banquinho de cócoras, cavalinho. A maioria recebeu ocitocina $(52,2 \%)$ e teve as membranas rompidas de forma artificial (51,2\%). Em grande parte delas, foi realizado tricotomia $(43,6 \%)$ e enema $(37,0 \%)$, e poucas receberam anestesia epidural ou ráqui $(9,1 \%)$. Durante o parto, pouquíssimas puderam escolher a posição diferente da litotômica para parir $(5,1 \%)$ e muitas receberam uma episiotomia $(44,1 \%)$ ou foi realizado pressão no fundo uterino para empurrar o bebê (manobra de Kristeller) (27,6\%). Poucas mulheres permaneceram na mesma sala durante o pré-parto, o parto e o pós-parto (sala PPP) (16,4\%) e menos da metade ficou em contato pele a pele com o bebê logo após o nascimento (43,3\%).

A Tabela 2 apresenta as prevalências e razões de prevalências brutas e ajustadas das práticas obstétricas realizadas durante o trabalho de parto, de acordo com a presença do acompanhante. A presença do acompanhante durante o trabalho de parto, após ajuste pelas variáveis idade, escolaridade, fonte de pagamento do parto, paridade e escore ABIPEME, esteve significativamente associada à maior oferta de líquidos ou alimentos $(\mathrm{RPa}=1,34$; IC95\% 1,10-1,63), à prescrição de algum tipo de dieta (RPa = 1,34 IC95\% 1,15-1,57), ao uso de MNF para alívio da dor $(\mathrm{RPa}=1,37$; IC95\% 1,21-1,56) e ao uso de amniotomia $(\mathrm{RPa}=1,10$; 
Tabela 1. Características sociodemográficas e obstétricas das mulheres que entraram em trabalho de parto. Região Sul do Brasil, 2011. $(n=2.070)$

\begin{tabular}{|c|c|c|c|}
\hline Variável & $\mathbf{n}$ & $\%$ & IC95\% \\
\hline \multicolumn{4}{|l|}{ Idade $(\mathrm{n}=2.070)$} \\
\hline $12-19$ anos & 461 & $22,{ }^{3}$ & $20,52-24,11$ \\
\hline $20-34$ anos & 1.404 & 67,8 & $65,78-69,81$ \\
\hline 35 anos ou mais & 205 & 9,9 & $8,69-11,27$ \\
\hline \multicolumn{4}{|l|}{ Escolaridade $(\mathrm{n}=2.064)$} \\
\hline Fundamental incompleto & 634 & 30,7 & $28,76-32,74$ \\
\hline Fundamental completo & 652 & 31,6 & $29,62-33,63$ \\
\hline Médio completo & 661 & 32,0 & $30,04-34,07$ \\
\hline Superior completo ou mais & 117 & 5,7 & $4,75-6,75$ \\
\hline \multicolumn{4}{|l|}{ Escore ABIPEME $(n=2.057)$} \\
\hline$A+B$ & 576 & 28,0 & $26,10-29,98$ \\
\hline $\mathrm{C}$ & 1.240 & 60,3 & $58,15-62,38$ \\
\hline $\mathrm{D}+\mathrm{E}$ & 241 & 11,7 & $10,39-13,18$ \\
\hline \multicolumn{4}{|l|}{ Paridade $(\mathrm{n}=2.069)$} \\
\hline Primípara & 890 & 43,0 & $40,89-45,16$ \\
\hline Multípara & 1.179 & 57,0 & $54,84-59,10$ \\
\hline \multicolumn{4}{|l|}{ Tipo de parto $(\mathrm{n}=2.070)$} \\
\hline Vaginal & 1.760 & 85,0 & $83,42-86,50$ \\
\hline Cesariana & 310 & 15,0 & $13,50-16,58$ \\
\hline \multicolumn{4}{|l|}{ Fonte pagamento parto $(n=2.070)$} \\
\hline Público & 1.857 & 89,7 & $88,32-90,95$ \\
\hline Privado & 213 & 10,3 & $9,05-11,67$ \\
\hline \multicolumn{4}{|l|}{ Presença do acompanhante } \\
\hline No trabalho de parto $(n=1.975)$ & 1.021 & 51,7 & $49,49-53,90$ \\
\hline No parto $(\mathrm{n}=1.759)$ & 693 & 39,4 & $37,14-41,70$ \\
\hline Na cesariana $(n=310)$ & 108 & 34,8 & $29,71-40,34$ \\
\hline \multicolumn{4}{|l|}{ Práticas trabalho de parto } \\
\hline Oferta de dieta $(n=1.938)$ & 354 & 18,2 & $16,57-20,01$ \\
\hline Prescrição de dieta $(n=1.374)$ & 463 & 32,7 & $30,30-35,19$ \\
\hline Livre movimentação ( $\mathrm{n}=1.893)$ & 1.124 & 59,2 & $57,02-61,44$ \\
\hline MNF alívio da dor $(\mathrm{n}=1.975)$ & 676 & 32,7 & $30,67-34,71$ \\
\hline Ocitocina $(n=1.875)$ & 1.008 & 52,2 & $49,97-54,42$ \\
\hline Amniotomia $(n=1.858)$ & 954 & 51,2 & $48,96-53,50$ \\
\hline Tricotomia $(n=1.875)$ & 843 & 43,6 & $41,46-45,88$ \\
\hline Enema $(n=1.875)$ & 714 & 37,0 & $34,85-39,15$ \\
\hline Analgesia de parto $(n=1.875)$ & 175 & 9,1 & $7,86-10,43$ \\
\hline \multicolumn{4}{|l|}{ Práticas no parto } \\
\hline Posição não litotômica & 90 & 5,1 & $4,19-6,27$ \\
\hline Episiotomia & 912 & 44,1 & $41,93-46,21$ \\
\hline Manobra de Kristeller & 571 & 27,6 & $25,76-29,62$ \\
\hline Sala PPP & 289 & 16,4 & $14,77-18,25$ \\
\hline Contato pele a pele & 889 & 43,3 & $41,21-45,50$ \\
\hline
\end{tabular}

ABIPEME: Classificação econômica preconizada pela Associação Brasileira de Empresas de Pesquisa; MNF: método não-farmacológico; PPP: pré-parto, parto e pós-parto

IC95\% 1,01-1,21) e de analgesia peridural ou ráqui ( $\mathrm{RPa}=1,84$; IC95\% 1,33-2,54). Além disso, a presença do acompanhante durante o trabalho de parto associou-se à redução no uso de tricotomia $(\mathrm{RPa}=0,59$; IC95\% 0,53-0,65) e de enema $(\mathrm{RPa}=0,49$; IC95\% 0,43-0,56).

A Tabela 3 apresenta as prevalências e razões de prevalências brutas e ajustadas das práticas obstétricas realizadas durante o parto, de acordo com a presença do acompanhante no momento do parto/nascimento. No momento do nascimento do bebê, a presença do acompanhante permaneceu significativamente associada à adoção pela mulher de posições diferentes da litotômica $(\mathrm{RP}=1,77$; IC95\% 1,16-2,72), à permanência na sala $\mathrm{PPP}(\mathrm{RP}=1,62$; IC95\% 1,31-2,00) e ao contato pele a pele entre a mãe e o bebê logo após o nascimento, tanto no parto (RP 1,81; IC95\% 1,64-1,99) quanto na cesariana (RP = 2,43; IC95\% 1,22-4,85). 
Tabela 2. Práticas obstétricas realizadas, durante o trabalho de parto, de acordo com a presença do acompanhante. Região Sul do Brasil, 2011. $(\mathrm{n}=2.070)$

\begin{tabular}{|c|c|c|c|c|c|c|c|c|}
\hline \multirow{3}{*}{ Variável } & \multicolumn{8}{|c|}{ Presença do acompanhante no trabalho de parto } \\
\hline & \multicolumn{2}{|c|}{ Sim } & \multicolumn{2}{|c|}{ Não } & \multirow{2}{*}{$\mathbf{R P}$} & \multirow{2}{*}{ IC95\% } & \multirow{2}{*}{$\mathrm{RPa}^{\mathrm{c}}$} & \multirow{2}{*}{ IC95\% } \\
\hline & $\mathbf{n}$ & $\%$ & $\mathbf{n}$ & $\%$ & & & & \\
\hline Oferta de dieta $(n=1.938)$ & 215 & 21,1 & 139 & $15,1^{\mathrm{a}}$ & 1,39 & $1,15-1,69^{a}$ & 1,34 & $1,10-1,63^{b}$ \\
\hline Prescrição de dieta $(n=1.374)$ & 275 & 37,5 & 177 & $27,7^{\mathrm{a}}$ & 1,35 & $1,16-1,58^{\mathrm{a}}$ & 1,34 & $1,15-1,57^{b}$ \\
\hline Livre movimentação $(\mathrm{n}=1.893)$ & 614 & 62,0 & 509 & $56,4^{a}$ & 1,10 & $1,02-1,19^{a}$ & 1,07 & $0,99-1,15$ \\
\hline MNF alívio da dor $(\mathrm{n}=1.975)$ & 411 & 40,2 & 265 & $27,8^{\mathrm{a}}$ & 1,45 & $1,28-1,64$ & 1,37 & $1,21-1,56^{\mathrm{b}}$ \\
\hline Ocitocina $(n=1.875)$ & 524 & 54,1 & 461 & $50,8)$ & 1,06 & $0,98-1,16$ & 1,05 & $0,96-1,15$ \\
\hline Amniotomia $(n=1.858)$ & 517 & 53,5 & 435 & $48,8^{\mathrm{a}}$ & 1,09 & $1,00-1,20^{\mathrm{a}}$ & 1,10 & $1,01-1,21^{\mathrm{b}}$ \\
\hline Tricotomia $(n=1.875)$ & 321 & 33,2 & 503 & $55,5^{a}$ & 0,60 & $0,54-0,66^{\mathrm{a}}$ & 0,59 & $0,53-0,65^{\mathrm{b}}$ \\
\hline Enema $(\mathrm{n}=1.875)$ & 247 & 25,5 & 453 & $49,9^{\mathrm{a}}$ & 0,51 & $0,45-0,58^{\mathrm{a}}$ & 0,49 & $0,43-0,56^{b}$ \\
\hline Analgesia de parto $(n=1.875)$ & 117 & 12,1 & 46 & $5,1^{\mathrm{a}}$ & 2,38 & $1,71-3,31^{\mathrm{a}}$ & 1,84 & $1,33-2,54^{\mathrm{b}}$ \\
\hline Parto vaginal $(n=1.975)$ & 889 & 87,1 & 853 & 89,4 & 0,97 & $0,94-1,00$ & 0,99 & $0,96-1,02$ \\
\hline
\end{tabular}

RP: razão de prevalência; RPa: razão de prevalência ajustada; MNF: método não-farmacológico; ABIPEME:

Classificação econômica preconizada pela Associação Brasileira de Empresas de Pesquisa

a Teste qui-quadrado: $\mathrm{p}<0,05$

${ }^{b}$ Teste de Wald: $\mathrm{p}<0,05$

c Ajustado por idade, escolaridade, pagamento do parto (público ou privado), paridade, e escore ABIPEME.

Tabela 3. Práticas obstétricas realizadas durante o parto, de acordo com a presença do acompanhante neste momento, nas mulheres que entraram em trabalho de parto. Região Sul do Brasil, 2011. ( $\mathrm{n}=2.324)$

\begin{tabular}{|c|c|c|c|c|c|c|c|c|}
\hline \multirow{3}{*}{ Variável } & \multicolumn{8}{|c|}{ Presença do acompanhante no parto } \\
\hline & \multicolumn{2}{|c|}{ Sim } & \multicolumn{2}{|c|}{ Não } & \multirow{2}{*}{$\mathbf{R P}$} & \multirow{2}{*}{ IC95\% } & \multirow{2}{*}{$\mathrm{RPa}^{\mathrm{c}}$} & \multirow{2}{*}{ IC95\% } \\
\hline & $\mathbf{n}$ & $\%$ & $\mathbf{n}$ & $\%$ & & & & \\
\hline \multicolumn{9}{|l|}{ No parto } \\
\hline Posição não litotômica $(n=1.753)$ & 49 & 7,1 & 41 & $3,9^{\mathrm{a}}$ & 1,83 & $1,22-2,74^{\mathrm{a}}$ & 1,77 & $1,16-2,72^{b}$ \\
\hline Episiotomia $(\mathrm{n}=1.759)$ & 393 & 56,7 & 519 & $48,7^{\mathrm{a}}$ & 1,16 & $1,06-1,27^{a}$ & 1,01 & $0,93-1,11$ \\
\hline Manobra de Kristeller $(\mathrm{n}=1.754)$ & 188 & 27,2 & 383 & $36,0^{\mathrm{a}}$ & 0,75 & $0,65-0,87^{a}$ & 0,67 & $0,58-0,78^{b}$ \\
\hline Sala PPP $(n=1.757)$ & 133 & 19,2 & 156 & $14,7^{\mathrm{a}}$ & 1,31 & $1,06-1,62^{\mathrm{a}}$ & 1,62 & $1,31-2,00^{b}$ \\
\hline Contato pele a pele $(n=1.741)$ & 456 & 66,1 & 390 & $37,1^{\mathrm{a}}$ & 1,78 & $1,62-1,96^{\mathrm{a}}$ & 1,81 & $1,64-1,99^{b}$ \\
\hline \multicolumn{9}{|l|}{ Na cesariana } \\
\hline Contato pele a pele $(n=309)$ & 27 & 25,0 & 16 & $8,0^{\mathrm{a}}$ & 3,14 & $1,77-5,57^{\mathrm{a}}$ & 2,43 & $1,22-4,85^{b}$ \\
\hline
\end{tabular}

RP: razão e prevalência; RPa: razão de prevalência ajustada; PPP: pré-parto, parto e pós-parto; ABIPEME:

Classificação econômica preconizada pela Associação Brasileira de Empresas de Pesquisa

${ }^{a}$ Teste qui-quadrado: $\mathrm{p}<0,05$

b Teste de Wald: $p<0,05$

${ }^{c}$ Ajustado por idade, escolaridade, pagamento do parto (público ou privado), paridade, e escore ABIPEME.

Além disso, a manobra de Kristeller foi significativamente menos realizada nas mulheres que estavam com o acompanhante $(\mathrm{RP}=0,67$; IC95\% 0,58-0,78).

A frequência de algumas intervenções que são consideradas de uso desnecessário ou inadequado, como a realização de episiotomia e o uso de ocitocina, não foram afetadas de forma significativa pela presença de acompanhante.

\section{DISCUSSÃO}

Os resultados mostram que a maioria das mulheres da região Sul não tem acesso às boas práticas na atenção ao parto e nascimento. A presença do acompanhante foi mais frequente no trabalho de parto do que no parto ou cesariana. Foram identificadas elevadas taxas de enema, tricotomia, manobra de Kristeller, amniotomia, ocitocina, episiotomia, e restrição hídrica e alimentar. Além disso, pouco mais da metade das parturientes teve liberdade de posição e movimento e raras foram as que conseguiram parir em posição não litotômica. A maioria delas não teve acesso ao uso de MNF para alívio da dor, à utilização de salas PPP e ao contato pele a pele com o recém-nascido logo após o parto. No entanto, a presença do acompanhante no trabalho de parto implicou em maior oferta de líquidos ou alimentos, prescrição de dieta, uso de MNF para alívio da dor e redução de tricotomia e enema. No parto, 
a presença do acompanhante associou-se à adoção de posição não litotômica, sala PPP, contato pele a pele e redução da manobra de Kristeller.

O fato de o acompanhante estar mais presente durante o trabalho de parto do que no momento do nascimento é semelhante aos achados de estudo realizado em Santa Catarina, no qual a permanência do acompanhante na sala de parto/cirúrgica não foi permitida na mesma proporção que nos outros locais do serviço obstétrico 5 . Essa restrição pode ser decorrente de que muitos profissionais não consideram o parto/nascimento como um evento familiar, mas sim um ato médico, que deve ser atendido em ambiente "estéril", sem a presença de pessoas leigas, devido ao seu potencial de risco e à possibilidade de intervenções caso sejam diagnosticadas intercorrências.

A tricotomia e o enema, realizados em quase metade das mulheres, são práticas prejudiciais e ineficazes, que deveriam ser desencorajadas e eliminadas por não apresentarem efeito benéfico significativo sobre as taxas de infecção ou deiscência da ferida perineal ou outras infecções neonatais ${ }^{2}$.

A manobra de Kristeller foi amplamente utilizada, apesar da inexistência de evidências suficientes que apoiem a sua recomendação, sendo semelhante a outros estudos brasileiros ${ }^{11,21}$. Além de proporcionar maior desconforto materno, essa manobra traz efeitos deletérios para o útero, o períneo e o feto e é ineficaz na redução do segundo estágio do trabalho de parto ${ }^{1,24}$.

Apesar de o parto ser um evento fisiológico, outras intervenções, como a infusão rotineira de ocitocina e a amniotomia, vêm sendo realizadas para prevenir o trabalho de parto prolongado. Neste estudo, metade das mulheres receberam pelo menos uma das duas intervenções, mesmo não havendo evidências suficientes que justifiquem essas práticas ${ }^{33}$.

Além dos riscos associados ao uso liberal da correção da dinâmica com ocitocina durante o trabalho de parto, incluindo exaustão materna, hiperestimulação do útero, ruptura do útero ou da placenta e sofrimento fetal ${ }^{22}$, a infusão endovenosa contínua também limita a liberdade de movimentação da mulher, podendo prolongar a duração do trabalho de parto. Em relação à amniotomia precoce, estudo de revisão concluiu que esse procedimento não deve ser recomendado de forma rotineira, devendo ser uma decisão discutida com as mulheres antes da sua realização ${ }^{36}$.

A elevada prevalência de episiotomia na América Latina ${ }^{6}$ também foi encontrada no presente estudo. A episiotomia caracteriza-se essencialmente como uma laceração de segundo grau, capaz de romper diversos grupamentos musculares e aumentar a ocorrência de lacerações de terceiro e quarto graus ${ }^{38}$. O ideal é que a taxa de episiotomia não ultrapasse $10 \%$, meta considerada razoável pela $\mathrm{OMS}^{30}$. Diversos países têm buscado uma política restritiva de realização de episiotomia. Nos EUA, o percentual caiu significativamente de 17,3\% em 2006 para 11,6\% em $2012^{13}$. Entretanto, ainda persistem elevadas taxas, em estudo realizado na Espanha, a taxa foi de $33,5 \%$ em $2011^{39}$ e em pesquisa realizada na Inglaterra, a taxa foi de $25 \%{ }^{32}$.

A minoria das mulheres estudadas recebeu algum tipo de dieta. Isso pode estar relacionado à resistência dos profissionais de saúde à ingesta hídrica e alimentar pela parturiente, apesar de as evidências científicas mostrarem efeitos deletérios do jejum no trabalho de parto ${ }^{c} \mathrm{e}$ de a OMS considerar o oferecimento de líquidos por via oral uma prática útil e que deve ser estimulada ${ }^{30}$.

Quase metade das mulheres estudadas não se movimentou durante o trabalho de parto, apesar da deambulação e as posições verticais reduzirem a dor e a duração desse período ${ }^{19}$.

${ }^{c}$ Amorim M. Assistência ao parto baseada em evidências. Rio de Janeiro; 2012 [citado 16 jun 2017]. Disponível em: http:// estudamelania.blogspot.com. br/2012/09/assistencia-ao-partobaseada-em.html
Além dessas várias intervenções, destaca-se que a maioria das mulheres não teve acesso aos MNF de alívio da dor, apesar da eficácia desses métodos? ${ }^{7}$ Esses resultados diferem de outros estudos com amostras menores, realizados em hospitais públicos da região Sul, nos quais as mulheres tiveram mais acesso à MNF, indicando que essas experiências ainda são pontuais e que dependem da filosofia assistencial ${ }^{5,16}$. 
O sistema PPP e o contato pele a pele na sala de parto ocorreram em menos da metade das parturientes. A sala PPP permite a presença de pessoas de escolha da parturiente e uma assistência humanizada e segura para o binômio mãe-bebê $\hat{~}^{37}$. O contato precoce pele a pele proporciona benefícios imediatos e em longo prazo, uma vez que melhora a efetividade da primeira mamada, regula a temperatura corporal do bebê e contribui para a vinculação materna ${ }^{27}$.

Nesta realidade extremamente intervencionista, a presença do acompanhante esteve associada à redução no uso de intervenções desnecessárias ou utilizadas de forma inadequada, seja no trabalho de parto, como o uso de amniotomia, tricotomia e enema, ou no parto, como a redução da manobra de Kristeller. Essas práticas não foram avaliadas na revisão sistemática atualizada em $2013^{17}$.

Além disso, a presença do acompanhante durante o trabalho de parto favoreceu a oferta de líquidos e alimentos e o uso de MNF para o alívio da dor. No parto, o acompanhante favoreceu a adoção de posição não litotômica, o atendimento da mulher no sistema PPP e o contato pele a pele logo após o nascimento. Nas mulheres que foram submetidas à cesariana, o contato pele a pele precoce ocorreu 2,4 vezes mais quando o acompanhante estava presente. A realização dessas boas práticas, respaldadas pelas evidências científicas, também não foram avaliadas na revisão sistemática sobre apoio no nascimento ${ }^{17}$.

Esses achados podem estar relacionados à filosofia assistencial implementada nas instituições de saúde, uma vez que, ao permitir o acompanhante, outras práticas também são reconhecidas como benéficas e passam a fazer parte da rotina, conforme já mencionado em outras pesquisas que retratam experiências locais no Brasil ${ }^{5,18}$. Além disso, quando o acompanhante é acolhido e estimulado a desenvolver ações de conforto físico, ele participa da aplicação de $\mathrm{MNF}^{16}$ e exerce o papel de intermediação, negociando os desejos da mulher com a equipe de saúde ${ }^{17}$.

Destaca-se que, no Brasil, as instituições de saúde que permitem a presença do acompanhante são as que buscam reduzir intervenções desnecessárias, sem evidências e não recomendadas pela OMS para a assistência ao parto, e que implementaram mudanças mínimas na ambiência e no mobiliário, tais como ter cadeiras para todos os acompanhantes ${ }^{5,9,10}$. Assim, a co-relação entre a presença do acompanhante e a adoção de boas práticas nas instituições pode ter contribuído para esses resultados. Também há de se considerar que o profissional de saúde tende a mudar de atitude quando o acompanhante está presente, o que pode ser observado nos estudos que mostram que as mulheres ficam mais satisfeitas com a experiência do parto e com as orientações e os cuidados recebidos dos profissionais de saúde quando possuem um acompanhante de sua escolha ${ }^{3,4,28}$.

Apesar dos benefícios apresentados, após ajuste pelas variáveis de controle, a presença do acompanhante na região Sul não apresentou qualquer impacto sobre a livre movimentação, o tipo de parto e as taxas de episiotomia e de ocitocina.

A presença do acompanhante pode não ter reduzido a prescrição de ocitocina e a realização de episiotomia por serem práticas utilizadas de forma rotineira em muitas instituições, muitas vezes sem ciência ou autorização da parturiente, reforçando ainda mais um modelo tecnocrático e mecanicista de assistência ao parto. Esses achados assemelham-se aos encontrados em revisão sistemática ${ }^{17}$, em que o apoio não teve impacto sobre a ocorrência de ocitocina e episiotomia. Em um estudo qualitativo, mulheres que sofreram episiotomia desconheciam a finalidade do procedimento e relataram que não foram solicitadas a autorizar o procedimento ${ }^{14}$.

Estudos internacionais buscam identificar os motivos que dificultam a apropriação das intervenções baseadas em evidências já conhecidas e da mudança na prática assistencial em todo o mundo, inclusive de práticas que poderiam ser utilizadas em locais com poucos recursos. Dentre as razões, destaca-se a falta de acesso ao conhecimento científico para profissionais dos países em desenvolvimento, bem como a falta de interesse de profissionais e gestores de serviços de saúde na produção da atenção obstétrica e neonatal de melhor qualidade ${ }^{8,12,34}$. 
Nesse estudo, a analgesia peridural ou ráqui foi mais frequente nas mulheres com acompanhante. Esse resultado difere do encontrado em revisão sistemática, em que o apoio reduziu a necessidade de analgesia ${ }^{17}$. Apesar de ser um procedimento que interfere na evolução do trabalho de parto, o resultado do presente estudo pode estar relacionado à inversão de conceito presente na realidade obstétrica brasileira, onde o excesso de intervenções muitas vezes é considerado sinônimo de cuidado.

Importante destacar que a maioria das variáveis analisadas por este estudo foram selecionadas do questionário de entrevista com a puérpera, sendo autorreferidas e, portanto, susceptíveis a viés de compreensão.

Outra limitação está relacionada ao desenho do estudo, ou seja, por ser um estudo transversal, apenas estimou a associação da presença do acompanhante com as boas práticas na assistência ao parto, mas não é capaz de indicar causalidade. Assim, é necessário considerar que, em algumas instituições, a associação existente pode ser decorrente do próprio modelo assistencial adotado, no qual a presença do acompanhante está inserida em um rol de boas práticas já realizadas. A Diretriz Nacional de Assistência ao Parto Normal, publicada em 2016, destaca que o apoio contínuo por acompanhante de escolha da mulher não dispensa o apoio oferecido pelo pessoal do hospital, a partir da adoção das melhores práticas disponíveis ${ }^{26}$.

O apoio contínuo proporcionado pelo acompanhante durante o trabalho de parto/parto, caracteriza-se como fator protetor ao favorecer a redução de intervenções e práticas danosas e agressivas em um momento em que a parturiente e o recém-nascido encontram-se extremamente vulneráveis às rotinas hospitalares e às decisões dos profissionais. Desta forma, é imprescindível a adequação das maternidades para incluir e acolher o acompanhante. Em relação ao uso de recursos necessários para garantir o apoio contínuo, destaca-se que o apoio por acompanhante de escolha e da própria rede social da mulher, a partir dos benefícios já descritos, poderia favorecer uma economia de recursos no número de profissionais necessários para garantir este apoio permanente ${ }^{26}$, ao que poderia ser destinado para a adequação estrutural das instituições à presença do acompanhante.

Para assegurar a presença do acompanhante de livre escolha da mulher, é fundamental que ocorra a fiscalização dos órgãos responsáveis nas instituições de saúde vinculadas ao SUS ou à saúde suplementar, bem como mudanças na formação dos profissionais para a assistência ao parto. No debate atual sobre as políticas públicas na atenção ao parto, é indispensável a garantia desse direito, que historicamente tem sido pauta do movimento de mulheres.

As mulheres também devem ser estimuladas, via redes sociais e grupos organizados, a lutar por uma assistência digna e humanizada, que resgate o protagonismo feminino e vislumbre o parto como um evento fisiológico e familiar.

Considerando o contexto brasileiro, no qual o acompanhante de escolha da mulher tem sido considerado o provedor de apoio, são necessários novos estudos que explorem outros resultados advindos da sua presença, ampliando o conhecimento acerca do seu papel no parto e nascimento.

\section{REFERÊNCIAS}

1. Api O, Balcin ME, Ugurel V, Api M, Turan C, Unal O. The effect of the uterine fundal pressure on the duration of the second stage of labor: a randomized controlled trial. Acta Obstet Gynecol Scand. 2009;88(3):320-4. https://doi.org/10.1080/00016340902730326

2. Basevi V, Lavender T. Routine perineal shaving on admission in labor. Cochrane Database Syst Rev. 2014;(11):CD001236. https://doi.org/10.1002/14651858.CD001236.pub2

3. Brüggemann OM, Parpinelli MA, Osis MJD, Cecatti JG, Carvalhinho Neto AS. Support to woman by a companion of her choice during childbirth: a randomized controlled trial. Reprod Health. 2007;4:5. https://doi.org/10.1186/1742-4755-4-5 
4. Brüggemann OM, Osis MJD, Parpinelli MA. Apoio no nascimento: percepções de profissionais e acompanhantes escolhidos pela mulher. Rev Saude Publica. 2007;41(1):44-52. https://doi.org/10.1590/S0034-89102006005000015 acompanhante de parto nos serviços públicos de saúde de Santa Catarina, Brasil. Esc Anna Nery. 2013;17(3):432-8. https://doi.org/10.1590/S1414-81452013000300005

6. Carroli G, Mignini L. Episiotomy for vaginal birth. Cochrane Database Syst Rev. 2009;(1):CD000081. https://doi.org/10.1002/14651858.CD000081.pub2

7. Cluett ER, Burns E. Immersion in water in labour and birth. Cochrane Database Syst Rev. 2009;2:CD000111. https://doi.org/10.1002/14651858.CD000111.pub3

8. Conde-Agudelo A, Rosas-Bermudez A, Gülmezoglu AM. Evidence-based intrapartum care in Cali, Colombia: a quantitative and qualitative study. BJOG. 2008;115(12):1547-56. https://doi.org/10.1111/j.1471-0528.2008.01930.x

9. Davim RMB, Torres GV, Dantas JC, Melo ES, Paiva CP, Vieira D, et al. Banho de chuveiro como estratégia não farmacológica no alívio da dor de parturientes. Rev Eletr Enf. 2008 [citado 16 jun 2017];10(3):600-9. Disponível em: http://www.fen.ufg.br/revista/v10/n3/v10n3a06.htm

10. Diniz CSG, D'Orsi E, Domingues RMSM, Torres JA, Dias MAB, Schneck CA, et al. Implementação da presença de acompanhantes durante a internação para o parto: dados da pesquisa nacional Nascer no Brasil. Cad Saude Publica. 2014;30 Supl 1:S140-53. https://doi.org/10.1590/0102-311X00127013

11. D'Orsi E, Chor D, Giffin K, Angulo-Tuesta A, Barbosa GP, Gama AS, et al. Qualidade da atenção ao parto em maternidades do Rio de Janeiro. Rev Saude Publica. 2005;39(4):645-54. https://doi.org/10.1590/S0034-89102005000400020

12. Fauveau V, Bernis L. "Good obstetrics" revisited: too many evidence-based practices and devices are not used. Int J Gynaecol Obstet. 2006;94(2):179-84. https://doi.org/10.1016/j.ijgo.2006.05.020

13. Friedman AM, Ananth CV, Prendergast E, D'Alton ME, Wright JD. Variation in and factors associated with use of episiotomy. JAMA. 2015;313(2):197-9. https://doi.org/10.1001/jama.2014.14774

14. Frigo J, Cagol G, Zocche DA, Zanotelli SS, Rodrigues RM, Ascari RA. Episiotomia: (des)conhecimento sobre o procedimento sob a ótica da mulher. BJSCR. 2014 [citado 16 jun 2017];6(2):5-10. Disponível em: http://www.mastereditora.com.br/ periodico/20140403_200543.pdf

15. Gama SGN, Viellas EF, Schilithz AOC, Theme Filha MM, Carvalho ML, Gomes KRO, et al. Fatores associados à cesariana entre primíparas adolescentes no Brasil, 2011-2012. Cad Saude Publica. 2014;30 Supl 1:S117-27. https://doi.org/10.1590/0102-311X00145513

16. Gayeski ME, Brüggemann OM, Monticelli M, Santos EKA. Application of nonpharmacologic methods to relieve pain during labor: the point of view of primiparous women. Pain Manag Nurs. 2015;16(3):273-84. https://doi.org/10.1016/j.pmn.2014.08.006

17. Hodnett ED, Gates S, Hofmeyr GJ, Sakala C. Continuous support for women during childbirth. Cochrane Database Syst Rev. 2013;(7):CD003766. https://doi.org/10.1002/14651858.CD003766. pub5

18. Lansky S. Gestão da qualidade e da integralidade do cuidado em saúde para a mulher e a criança no SUS - BH: a experiência da comissão perinatal. Rev Tempus Actas Saude Coletiva. 2010;4(4):191-9.

19. Lawrence A, Lewis L, Hofmeyr GJ, Dowswell T, Styles C. Maternal positions and mobility during first stage labour. Cochrane Database Syst Rev. 2009;(2):CD003934. https://doi.org/10.1002/14651858.CD003934.pub2

20. Leal MC, Silva AAM, Dias MAB, Gama SGN, Rattner D, Moreira ME, et al. Birth in Brazil: national survey into labour and birth. Reprod Health. 2012;9:15. https://doi.org/10.1186/1742-4755-9-15

21. Leal MC, Pereira APE, Domingues RMSM, Theme Filha MM, Dias MAB, Nakamura-Pereira M, et al. Intervenções obstétricas durante o trabalho de parto e parto em mulheres brasileiras de risco habitual. Cad Saude Publica. 2014;30 Supl 1:S17-32. https://doi.org/10.1590/0102-311X00151513

22. Lovold A, Stanton C. Use of oxytocin and misoprostol for induction or augmentation of labor in low - resource settings: a working paper review. Washington (DC): Department of Population, Family and Reproductive Health; 2008 [citado 16 jun 2017]. Disponível em: http://www. pphprevention.org/files/Induction_Augmentationworkingpaper

23. Maia MB. Humanização do parto: políticas públicas, comportamento organizacional e ethos profissional. Rio de Janeiro: Editora Fiocruz; 2010. 
24. Matsuo K, Shiki Y, Yamasaki M, Shimoya K. Use of uterine fundal pressure maneuver at vaginal delivery and risk of severe perineal laceration. Arch Gynecol Obstet. 2009;280(5):781-86. https://doi.org/10.1007/s00404-009-1015-2

25. Ministério da Saúde (BR). Portaria № 1459, de 24 de junho de 2011. Institui, no âmbito do Sistema Único de Saúde - SUS-, a Rede Cegonha. Brasília (DF); 2011 [citado 16 jun 2017]. Disponível em: http://bvsms.saude.gov.br/bvs/saudelegis/gm/2011/prt1459_24_06_2011.html

26. Ministério da Saúde (BR), Secretaria de Ciência, Tecnologia e Insumos Estratégicos. Diretriz Nacional de Assistência ao Parto Normal: relatório de recomendação. Brasília (DF): CONITEC; 2016 [citado 16 jun 2017]. Disponível em: http://conitec.gov.br/images/Consultas/2016/Relatorio_DiretrizPartoNormal_CP.pdf

27. Moore ER, Anderson GC, Bergman N, Dowswell. Early skin-to-skin contact for mothers and their healthy newborn infants. Cochrane Database Syst Rev. 2012;(5):CD003519. https://doi.org/10.1002/14651858.CD003519.pub3

28. Morhason-Bello IO, Adedokun BO, Ojengbede OA, Olayemi O, Oladokun A, Fabamwo AO. Assessment of the effect of psychosocial support during childbirth in Ibadan, south-west Nigeria: a randomised controlled trial. Aust N Z J Obstet Gynaecol. 2009;49(2):145-50. https://doi.org/10.1111/j.1479-828X.2009.00983.x

29. Motta CCL, Crepaldi MA. O pai no parto e apoio emocional: a perspectiva da parturiente. Paidéia (Ribeirão Preto). 2005;15(30):105-18. https://doi.org/10.1590/S0103-863X2005000100012

30. Organização Mundial da Saúde, Saúde Reprodutiva e da Família, Unidade de Maternidade Segura, Saúde Materna e Neonatal. Assistência ao parto normal: um guia prático. Brasília (DF): Ministério da Saúde; 1996.

31. Perdomini FRI, Bonilha ALL. A participação do pai como acompanhante da mulher no parto. Texto Contexto Enferm. 2011;20(3):445-52. https:doi.org/10.1590/S0104-07072011000300004

32. Redshaw M, Heikkila K. Delivered with care: a national survey of women's experience of maternity care 2010. Oxford (UK): University of Oxford, National Perinatology Epidemiology Unity; 2010

33. Schmitz T, Meunier E. Mesures à prendre pendant le travail pour réduire le nombre d'extractions instrumentales. J Gynecol Obstet Biol Reprod (Paris). 2008;37 Suppl 8:S179-87. https://doi.org/10.1016/S0368-2315(08)74756-2

34. SEA-ORCHID Study Group, Laopaiboon M, Lumbiganon P, Mcdonald SJ, Henderson-Smart DJ, Green S, Crowther CA, et al. Use of evidence-based practices in pregnancy and childbirth: South East Asia Optimising Reproductive and Child Health in Developing Countries Project. PLoS One. 2008;3(7):e2646. https://doi.org/10.1371/journal.pone.0002646

35. Silva LM, Barbieri M, Fustinoni SM. Vivenciando a experiência da parturição em um modelo assistencial humanizado. Rev Bras Enferm. 2011;64(1):60-5. https://doi.org/10.1590/S0034-71672011000100009

36. Smith RMD, Markham C, Dowswell T. Amniotomy for shortening spontaneous labour. Cochrane Database Syst Rev. 2013;(6):CD006167. https://doi.org/10.1002/14651858.CD006167.pub4

37. Stancato K, Vergílio MSTG, Bosco CS. Avaliação da estrutura e assistência em sala de pré-parto, parto e pós-parto imediato-PPP de um hospital universitário. Cienc Cuid Saude. 2011;10(3):5418. https://doi.org/10.4025/cienccuidsaude.v10i3.12656

38. Steiner N, Weintraub AY, Wiznitzer A, Sergienko R, Sheiner E. Episiotomy: the final cut? Arch Ginecol Obstet. 2012;286(6):1369-73. https://doi.org/10.1007/s00404-012-2460-x

39. Valero SP. Episiotomía en partos vaginales eutócicos en el Hospital Universitario "La Ribera". Nure Investig. 2013 [citado 20 jun 2017];(63):1-6. Disponível em: http://www.nureinvestigacion. es/OJS/index.php/nure/article/view/620/609

40. Vasconcellos MTL, Silva PLN, Pereira APE, Schilithz AOC, Souza Junior PRB, Szwarcwald CL. Desenho da amostra Nascer no Brasil: Pesquisa Nacional sobre Parto e Nascimento. Cad Saude Publica. 2014;30 Supl 1:49-58. https://doi.org/10.1590/0102-311X00176013

Financiamento: Conselho Nacional de Desenvolvimento Científico e Tecnológico (CNPq - Processo 57/2009; bolsa produtividade para d'Orsi E - Processo 304606/2016-2 e Brüggemann OM - Processo 304970/2016-6).

Contribuição dos Autores: Concepção e planejamento do estudo: JJCM, OMB. Coleta, análise e interpretação dos dados: JJCM, OMB, ED, PFF. Elaboração ou revisão do manuscrito: JJCM, OMB. Aprovação da versão final: JJCM, OMB, ED. Responsabilidade pública pelo conteúdo do artigo: JJCM, OMB, ED, PFF.

Conflito de Interesses: Os autores declaram não haver conflito de interesses. 